\title{
Low-grade endometrial stromal sarcoma in a young woman diagnosed after resection of endometrial polyp-like lesions: A case report
}

\author{
HITOMI MATSUKAWA, NAMI OTA, KAHO NISHIOKA, TOMOKO NOGUCHI, NAOYUKI IWAHASHI, \\ YASUSHI MABUCHI, SHIGETAKA YAGI, SAWAKO MINAMI and KAZUHIKO INO
}

Department of Obstetrics and Gynecology, Wakayama Medical University, Wakayama 641-0012, Japan

Received April 26, 2021; Accepted August 5, 2021

DOI: $10.3892 / \mathrm{mco} .2021 .2400$

\begin{abstract}
Low-grade endometrial stromal sarcoma (LG-ESS) is a rare tumor that mostly occurs in perimenopausal women. Treatment with total hysterectomy and bilateral salpingo-oophorectomy is recommended, although fertility preservation or ovarian preservation may be considered in younger patients. The present study reports a case of LG-ESS in a young woman diagnosed after resection of endometrial polyp-like lesions. A 26-year-old nulligravid woman was referred to our hospital after being diagnosed with endometrial polyps. Hysteroscopic endometrial polypectomy was performed twice, and LG-ESS was suspected on postoperative pathological examination. Magnetic resonance imaging revealed a tumor $5-\mathrm{cm}$ in diameter on the right side of the uterus. In light of the young age of the patient, tumorectomy was first performed, and postoperative pathological diagnosis was LG-ESS with the positive resection margin. After thorough discussion with the patient about fertility preservation and recurrence risk, a total abdominal hysterectomy and ovarian preservation was performed. Medroxyprogesterone therapy was performed postoperatively and no recurrence was observed for 2 years.
\end{abstract}

\section{Introduction}

Low-grade endometrial stromal sarcoma (LG-ESS) is a rare tumor accounting for less than $1 \%$ of uterine malignancies $(1,2)$. Total hysterectomy and bilateral adnexectomy with/without lymphadenectomy are recommended for treatment of LG-ESS, and hormone therapy is also recommended as a treatment option for patients with advanced or recurrent

Correspondence to: Professor Kazuhiko Ino, Department of Obstetrics and Gynecology, Wakayama Medical University, 811-1 Kimiidera, Wakayama 641-0012, Japan

E-mail:kazuino@wakayama-med.ac.jp

Key words: low-grade endometrial stromal sarcoma, fertilitysparing treatment, pathological diagnosis, hormone therapy disease (3). Patients with stage I-II disease have a relatively favorable prognosis $(2,3)$. This tumor is most often seen in women in their 40 to $50 \mathrm{~s}$, but fertility-sparing surgery and ovarian preservation may be considered in younger patients. However, reports on fertility-sparing treatment for LG-ESS are very limited and no such treatment has been established. Herein, we report a case of LG-ESS in a woman in her 20s who was diagnosed with the disease after endometrial polyp-like lesions were removed. After tumor resection, she underwent total hysterectomy and ovarian preservation, followed by high-dose progesterone therapy. No recurrence has been detected to date. We also reviewed some important reports on fertility-sparing management of LG-ESS in the literature.

\section{Case report}

The patient was a 26-year-old, unmarried, nulligravid woman with no particular medical or family history. She visited a local hospital with a chief complaint of atypical genital bleeding, and was referred to our hospital after an intrauterine mass measuring $2 \mathrm{~cm}$ was pointed out. At the time of the first visit to our department, the abdomen was soft, and speculum examination showed no clear abnormalities. The uterus was the size of a hen egg on pelvic examination, and the bilateral adnexa were not palpated. The endometrium was as thickened as $15 \mathrm{~mm}$ on transvaginal ultrasonography, but there were no abnormal findings in the myometrium (Fig. 1A). Hysteroscopy revealed a $1.5-\mathrm{cm}$ polyp-like mass in the intrauterine cavity. Endometrial cytology test was negative. A blood test showed anemia with a hemoglobin level of $9.6 \mathrm{~g} / \mathrm{dl}$, but the tumor markers were within the normal ranges [CA125: $16.9 \mathrm{U} / \mathrm{ml}$ (reference value: $\leq 31.4 \mathrm{U} / \mathrm{ml}$ ); CA19-9: $7.3 \mathrm{U} / \mathrm{ml}$ (reference value: $\leq 37 \mathrm{U} / \mathrm{ml}$ )]. Based on these findings, endometrial polyps were suspected, and hysteroscopic polypectomy was performed. Pathological examination of the resected tissue was compatible with endometrial polyps with hemorrhagic granulation tissue-like tissue. It was decided that the patient should be followed up because no malignant findings were observed. Two months after hysteroscopic surgery, however, she was referred again by the local hospital with chief complaints of atypical genital bleeding and recurrence of intrauterine lesions, and hysteroscopy revealed a new smooth polyp-like mass $1 \mathrm{~cm}$ in 
diameter in the intrauterine cavity (Fig. 1B). Because recurrence of endometrial polyps was suspected, hysteroscopic polypectomy was performed once again. Pathological examination of the resected tissue showed overgrowth of endometrial stromal cells, and neoplastic changes were not ruled out. Accordingly, LG-ESS was included in the differential diagnosis, although clear atypical cells were not observed (Fig. 1C). The patient was closely examined for tumor invasion and distant metastasis because the possibility of a malignant tumor was not precluded. T2-weighted and diffusion-weighted magnetic resonance imaging (MRI) revealed a $4.5-\mathrm{cm}$ high-intensity area on the right side of the uterus (Fig. 2A and B), and positron emission tomography/computed tomography (PET/CT) showed an accumulation of fluorodeoxyglucose at the same site with an SUVmax of 4.9 (Fig. 2C). There were no findings suggestive of metastasis. Surgical treatment was proposed based on these examination results, but the patient strongly wished to preserve fertility. It was therefore decided to perform enucleation of the uterine mass through laparotomy, which we expected would also assist in confirming the diagnosis. Intraoperative findings indicated that the mass, which had an indistinct margin, protruded into the intrauterine cavity in the form of a polyp and deeply invaded the myometrium as well. Cytology of peritoneal lavage was negative. A 54-g mass $5 \mathrm{~cm}$ in size was surgically removed. The pathological findings of the excised mass showed atypical cells similar to endometrial stromal cells proliferating and invading the myometrium without distinct borders, and immunostaining was positive for CD10, estrogen receptor (ER), and progesterone receptor (PR). A diagnosis of LG-ESS was made (Fig. 3A-C). Because the surgical margin was positive, the patient was further examined for residual disease and metastasis in order to determine the subsequent treatment strategy. No residual tumor was evident in the uterus on MRI, and contrast-enhanced CT also showed no lymph node metastasis or distant metastasis. Based on these examination results, we made a diagnosis of stage IB LG-ESS. In light of the fact that the patient was as young as 26 years old and she was an unmarried, nulligravid woman, the possibility of fertility preservation and the attendant recurrence risk were fully explained, including that future possibility of a surrogate pregnancy or uterus transplantation remains even if she makes a choice of hysterectomy After that, she strongly desired hysterectomy and ovarian preservation, and total abdominal hysterectomy and bilateral salpingectomy were performed, with both ovaries being preserved. The pathological diagnosis of the excised uterus was LG-ESS, pT1b v(+), ly(+), and the resection margin was negative. Generally, follow-up observation without adjuvant therapy may be considered for patients with stage I LG-ESS who have undergone complete removal of the lesions. In the present case, however, high-dose progesterone therapy [medroxyprogesterone acetate (MPA), $600 \mathrm{mg} /$ day] was given from 1 month after surgery for 1 year in consideration of recurrence risk because the ovaries were preserved. Two years have elapsed since the surgery, and no recurrence has been observed to date.

\section{Discussion}

In the present case, the diagnosis of LG-ESS was made after the patient underwent surgery to excise endometrial polyp-like lesions twice. Because she was as young as 26 years old, fertility-sparing surgery including ovarian and uterine preservation was considered. The choice of operative procedure was not straightforward, but the patient first underwent tumor resection after due informed consent. She then underwent two-stage total hysterectomy, with the ovaries being preserved. Considering the risk of postoperative recurrence, we provided high-dose oral progesterone therapy. Two years have passed since the surgery, and no recurrence has been observed to date.

LG-ESS is common in women in their 40 and 50s, and patients with LG-ESS present with symptoms such as atypical genital bleeding, heavy menstrual bleeding, enlarged uterus, and lower abdominal pain (4). In general, a yellowish-white polyp-like mass is formed and often grows to fill the intrauterine cavity, but it may also invade the myometrium and vasculature and extend to the ovaries, pelvis, abdominal cavity, and retroperitoneal lymph nodes $(5,6)$. These characteristics are congruent with the fact that our patient had atypical genital bleeding as the initial symptom and that endometrial polyp-like lesions first appeared and then detected as a tumor invading the myometrium. According to previous reports, it is often difficult to distinguish LG-ESS from endometrial polyps or uterine fibroids preoperatively, and LG-ESS is sometimes diagnosed only after postoperative pathological examination $(5,6)$. In the present case, polyp-like lesions were confined to the intrauterine cavity when they were found, and there were no lesions in the myometrium on ultrasonography, so we did not perform MRI at this timing. The pathological findings at the time of the first polypectomy were not clearly malignant due to lack of cellular atypia. However, the polyp-like lesions recurred soon afterwards and grew further into the myometrium. It is therefore inferred that the initial polyp-like mass may have been an incipient lesion of LG-ESS. We speculate that the tumor was initially confined to the polypoid lesion within the uterine cavity at the time of the first hysteroscopic resection, but it had progressed rapidly and invaded into the myometrium at the second hysteroscopic resection; thus, it might have been better to examine the tumor extension using MRI before the second hysteroscopic polypectomy and to evaluate the safety and adequacy of the hysteroscopic resection.

Standard treatment for LG-ESS is hysterectomy and bilateral adnexectomy (3). Lymphadenectomy or lymph node biopsy is also recommended because $9-33 \%$ of this disease has pelvic node metastasis $(7,8)$, although its impact on survival is not clear. In our patient, it might have been better to have lymphadenectomy to decide the disease stage correctly, but we did not perform it because there was no suspicious finding of lymph node metastasis on PET/CT.

By contrast, management of LG-ESS in young patients who wish to preserve their fertility has not yet been established, with only a few reports available on the outcome of fertility-sparing treatment of LG-ESS (9-12) as summarized in Table I. Xie et al (9) studied 17 younger patients (aged 15-37 years) with stage I LG-ESS (6 stage IA patients and 11 stage IB patients) in whom laparoscopic/hysteroscopic or open tumor resection was performed with successful fertility preservation. In their study, 12 of the 17 patients received postoperative hormonal therapy. Recurrence of the disease was seen in 10 of the 

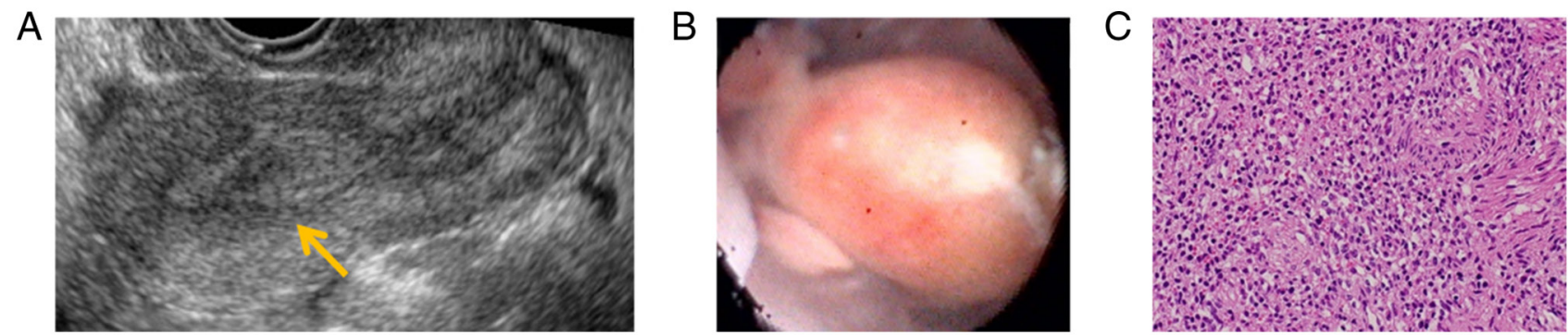

Figure 1. Images and pathological findings of endometrial polyp-like lesions at initial diagnosis. (A) Transvaginal ultrasonography finding at initial examination. The endometrium was thickened and a $15-\mathrm{mm}$ large polyp-like mass was observed in the lumen (arrow), but there were no abnormal findings in the myometrium. (B) Hysteroscopy revealed a smooth mass with a diameter of $\sim 1-\mathrm{cm}$ protruding into the intrauterine cavity. (C) Histopathological image at second polypectomy (hematoxylin-eosin staining; magnification, x200). Proliferation of single spindle-shaped endometrial stromal cells was evident, and although there was no obvious cellular atypia, malignant changes could not be ruled out.
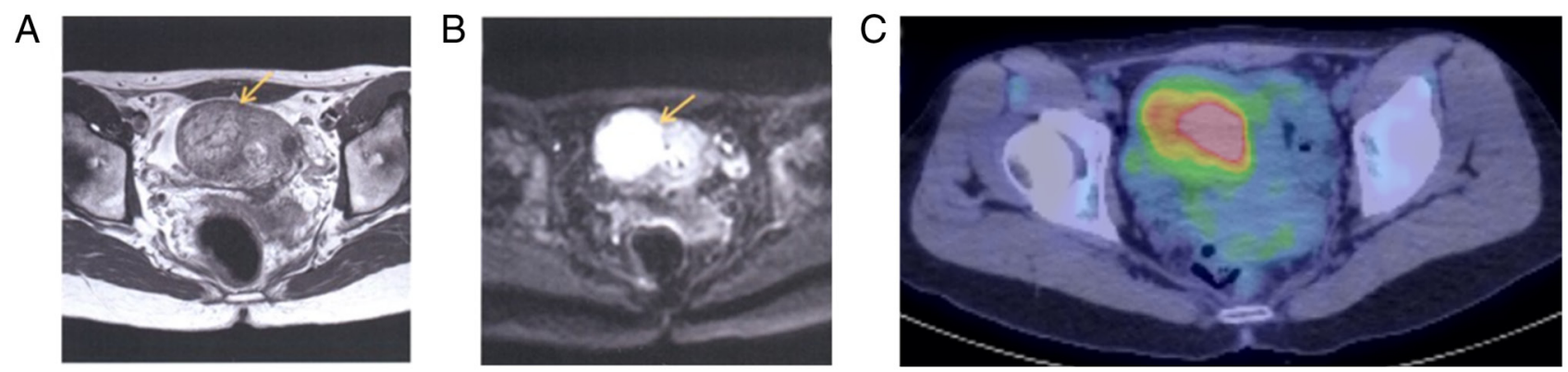

Figure 2. Magnetic resonance imaging and PET/CT of tumor. (A) T2-weighted image and (B) diffusion-weighted image. A high-intensity area of $~ 45$-mm in diameter was observed in the right corner of the uterus, suggesting invasion into the myometrium (indicated by arrows). (C) PET/CT revealed an accumulation of FDG on the right side of the uterus with an SUVmax of 4.9. There were no other obvious abnormal FDG accumulations suggestive of metastasis. PET/CT, positron emission tomography-computed tomography; FDG, fluorodeoxyglucose; SUVmax, maximum standardized uptake value.
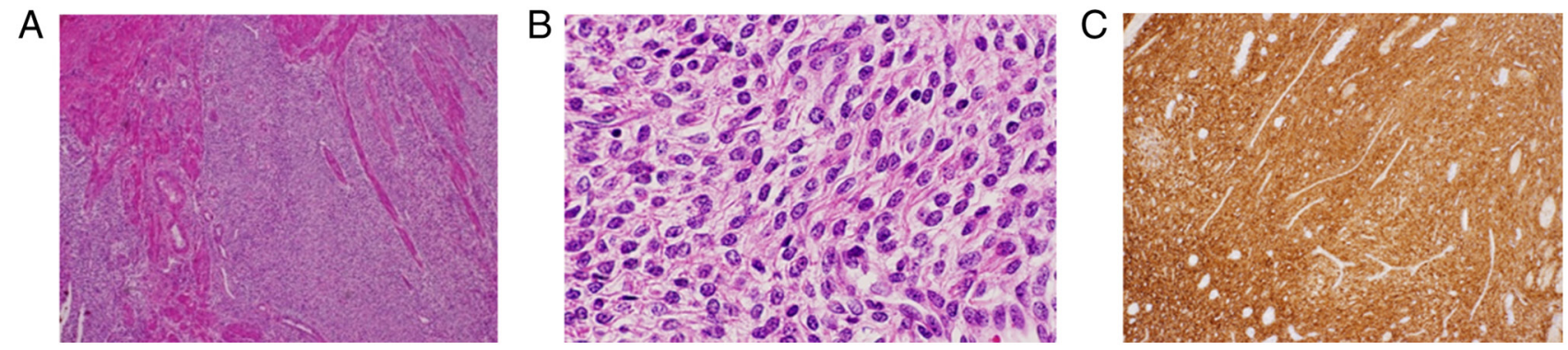

Figure 3. Histopathological images of the resected tumor. (A) Hematoxylin-eosin staining (magnification, x40). (B) Hematoxylin-eosin staining (magnification, x200). Atypical cells similar to endometrial stromal cells proliferated and invaded the myometrium without distinct borders. A diagnosis of low-grade endometrial stromal sarcoma was made. The resection margin was positive. (C) CD10 immunohistochemical staining of formalin-fixed paraffin-embedded tumor sections using anti-CD10 monoclonal antibody (Clone, 56C6; magnification, x40). The tumor cells were diffusely positive for CD10.

17 patients (59\%), but subsequent treatment resulted in a favorable outcome, with no mortality being reported in any patient. Furthermore, five of eight patients who wished to become pregnant succeeded in giving birth. Similarly, Laurelli et al (10) reported that six stage IA LG-ESS patients aged 18 to 40 years who had polypoid or submucosal tumors in the intrauterine cavity underwent hysteroscopic tumor resection, and five of them were treated with hormonal therapy with megestrol acetate (MA) for 1 to 2 years. All of these patients survived without recurrence, and three patients became pregnant. Jin et al (11) also reported that five LG-ESS patients aged 28 to 37 years who wished to preserve their fertility underwent local tumor resection and adjuvant hormonal therapy.
Although one patient had recurrent disease and subsequently underwent total hysterectomy, all five patients were alive, and three of them became pregnant and gave birth. They recommend that hormonal therapy should be given for 6 months after fertility-sparing surgery. By contrast, a retrospective analysis of 153 LG-ESS patients by Bai et al (12) showed that the recurrence rate was as high as $79 \%$ (15 of 19 patients) in patients who underwent tumor enucleation alone at the initial surgery, and that uterine preservation with tumor enucleation alone, ovarian preservation, and positive surgical margins were the three independent prognostic factors for recurrence. However, ovarian preservation and tumor enucleation did not significantly affect the overall survival. Based on these results, 
Table I. Summary of the reports on fertility-sparing treatment for young patients with low-grade endometrial stromal sarcoma.

\begin{tabular}{|c|c|c|c|c|c|c|c|c|c|}
\hline $\begin{array}{l}\text { Author, } \\
\text { year }\end{array}$ & $\begin{array}{c}\text { Case } \\
\text { no. }\end{array}$ & $\begin{array}{l}\text { Age, } \\
\text { years }\end{array}$ & Stage & $\begin{array}{l}\text { Surgical } \\
\text { resection } \\
\text { procedure }\end{array}$ & $\begin{array}{l}\text { Adjuvant } \\
\text { hormone } \\
\text { therapy }\end{array}$ & Recurrence & Pregnancy & $\begin{array}{l}\text { Final } \\
\text { clinical } \\
\text { outcome }\end{array}$ & (Refs.) \\
\hline $\begin{array}{l}\text { Xie et al, } \\
2017\end{array}$ & 17 & $15-37$ & $\begin{array}{l}\text { IA:6 } \\
\text { IB: } 11\end{array}$ & $\begin{array}{l}\text { Laparotomy:5 } \\
\text { Laparoscopy:7 } \\
\text { Hysteroscopy:5 }\end{array}$ & $\begin{array}{l}\text { MPA:3 } \\
\text { MA:5 } \\
\text { GnRHa:2 } \\
\text { GnRHa/LNG:2 } \\
\text { None:5 }\end{array}$ & 10 & 5 & NED:17 & (9) \\
\hline $\begin{array}{l}\text { Laurelli et al, } \\
2015\end{array}$ & 6 & $18-40$ & IA:6 & Hysteroscopy: 6 & $\begin{array}{l}\text { MA:5 } \\
\text { None:1 }\end{array}$ & 0 & 3 & NED:6 & (10) \\
\hline $\begin{array}{l}\text { Jin et al, } \\
2015\end{array}$ & 5 & $28-37$ & $\begin{array}{l}\text { IA:3 } \\
\text { IB:2 }\end{array}$ & Laparoscopy:5 & $\begin{array}{l}\text { MA:4 } \\
\text { GnRHa:1 }\end{array}$ & 1 & 3 & NED:5 & (11) \\
\hline $\begin{array}{l}\text { Bai et al, } \\
2014\end{array}$ & 19 & $\begin{array}{c}\text { Not } \\
\text { described }\end{array}$ & $\begin{array}{c}\text { Not } \\
\text { described }\end{array}$ & $\begin{array}{l}\text { Not described } \\
\text { in detail }\end{array}$ & $\begin{array}{l}\text { Not described } \\
\text { in detail }\end{array}$ & 15 & 5 & $\begin{array}{l}\text { NED: } 18 \\
\text { DOD: } 1\end{array}$ & (12) \\
\hline
\end{tabular}

MPA, medroxyprogesterone acetate; MA, megestrol acetate; GnRHa, gonadotropin-releasing hormone analogues; LNG, levonorgestrel-releasing intrauterine device; NED, no evidence of disease; DOD, death of disease.

Bai et al (12) suggested that tumor enucleation for LG-ESS should be performed after adequate informed consent only in patients with a strong desire for fertility preservation, and total hysterectomy should be recommended after the completion of pregnancy and delivery.

Since positive resection margins can be a prognostic factor for recurrence (12), it is of importance that fertility-sparing surgery should be performed for polypoid lesions in the intrauterine cavity, and for cases with the presence of intramuscular involvement, the surrounding normal myometrium should also be excised. In our case, the patient strongly desired to preserve fertility, and tumor enucleation was performed accordingly. Later on, however, it was decided to perform two-stage total hysterectomy after careful consideration of the high risk of recurrence due to positive resection margins, although the ovaries were preserved according to her strong wish. Ovarian preservation has been reported to be associated with an increased recurrence rate in premenopausal women since the growth and development of LG-ESS is hormone-dependent (13-15). However, there are no definitive reports that ovarian preservation shortens overall survival (16), and the rights and wrongs of ovarian preservation should be thoroughly discussed with the patient.

Being highly sensitive to hormonal therapy, tumor cells in LG-ESS frequently express ER and PR $(5,6)$ and in most cases express aromatase as well (17). They are also characterized by diffuse CD10 positivity on immunostaining $(5,6)$. In keeping with the previous reports, ER, PR, and CD10 were strongly positive in our patient. MA is most commonly used in adjuvant hormonal therapy following fertility-sparing surgery (9-11), and other treatments, such as MPA (9), levonorgestrel-releasing intrauterine device (LNG-IUD) (9) and gonadotropin-releasing hormone analogues (GnRHa) (9), have been reported in several cases. In our case, MPA at a daily oral dose of $600 \mathrm{mg}$ with low-dose aspirin, which is widely used as a fertility-sparing therapy in young women with endometrial carcinoma in Japan (18), was administered for 1 year, and no recurrence has been observed after completion of hormonal therapy. Careful follow-up over a long period is warranted.

\section{Acknowledgements}

Not applicable.

\section{Funding}

No funding was received.

\section{Availability of data and materials}

The datasets used and/or analyzed during the current study are available from the corresponding author on reasonable request.

\section{Authors' contributions}

HM, NO and KI conceived and designed this case report. $\mathrm{HM}, \mathrm{NO}$ and KI wrote the initial draft of the report. KN, TN and NI collected the clinical data. YM, SY and SM analyzed the data from images and pathological examination. NO and KI confirm the authenticity of all the raw data. All authors have read and approved the final version of the manuscript.

\section{Ethics approval and consent to participate}

Written informed consent for surgery and tissue collection was obtained from the patient.

\section{Patient consent for publication}

Written informed consent for publication of the present report was obtained from the patient. 


\section{Competing interests}

The authors declare that they have no competing interests.

\section{References}

1. Hosh M, Antar S, Nazzal A, Warda M, Gibreel A and Refky B: Uterine sarcoma: Analysis of 13,089 cases based on surveillance, epidemiology and end results database. Int J Gynecol Cancer 26: 1098-1104, 2016.

2. Brooks SE, Zhan M, Cote T and Baquet CR: Surveillance, epidemiology, and end results analysis of 2677 cases of uterine sarcoma 1989-1999. Gynecol Oncol 93: 204-208, 2004.

3. Amant F, Coosemans A, Debiec-Rychter M, Timmerman D and Vergote I: Clinical management of uterine sarcomas. Lancet Oncol 10: 1188-1198, 2009.

4. Thiel FC and Halmen S: Low-grade endometrial stromal sarcoma-a review. Oncol Res Treat 41: 687-692, 2018.

5. Chang KL, Crabtree GS, Lim-Tan SK, Kempson RL and Hendrickson MR: Primary uterine endometrial stromal neoplasms. A clinicopathologic study of 117 cases. Am J Surg Pathol 14: 415-438, 1990.

6. Hoang L, Chiang S and Lee CH: Endometrial stromal sarcomas and related neoplasms: New developments and diagnostic considerations. Pathology 50: 162-177, 2018.

7. Leath CA III, Huh WK, Hyde J Jr, Cohn DE, Resnick KE, Taylor NP, Powell MA, Mutch DG, Bradley WH, Geller MA, et al: A multi-institutional review of outcomes of endometrial stromal sarcoma. Gynecol Oncol 105: 630-634, 2007.

8. Riopel J, Plante M, Renaud MC, Roy M and Têtu B: Lymph node metastasis in low-grade endometrial stromal sarcoma. Gynecol Oncol 96: 402-406, 2005

9. Xie W, Cao D, Yang J, Jiang X, Shen K, Pan L, Huang H, Lang J, You Y and Chen J: Fertility-sparing surgery for patients with low-grade endometrial stromal sarcoma. Oncotarget 8 : 10602-10608, 2017.
10. Laurelli G, Falcone F, Scaffa C, Messalli EM, Del Giudice M, Losito S and Greggi S: Fertility-sparing management of low-grade endometrial stromal sarcoma: Analysis of an institutional series and review of the literature. Eur J Obstet Gynecol Reprod Biol 195: 61-66, 2015.

11. Jin Y, Li Y, Deng CY, Tian QJ, Chen H and Pan LY: Fertility-sparing treatment of low-grade endometrial stromal sarcoma. Int J Clin Exp Med 8: 5818-5821, 2015.

12. Bai H, Yang J, Cao D, Huang H, Xiang Y, Wu M, Cui Q, Chen J, Lang $J$ and Shen K: Ovary and uterus-sparing procedures for low-grade endometrial stromal sarcoma: A retrospective study of 153 cases. Gynecol Oncol 132: 654-660, 2014.

13. Amant F, De Knijf A, Van Calster B, Leunen K, Neven P, Berteloot P, Vergote I, Van Huffel S and Moerman P: Clinical study investigating the role of lymphadenectomy, surgical castration and adjuvant hormonal treatment in endometrial stromal sarcoma. Br J Cancer 97: 1194-1199, 2007.

14. Berchuck A, Rubin SC, Hoskins WJ, Saigo PE, Pierce VK and Lewis JL Jr: Treatment of endometrial stromal tumors. Gynecol Oncol 36: 60-65, 1990.

15. Li N, Wu LY, Zhang HT, An JS, Li XG and Ma SK: Treatment options in stage I endometrial stromal sarcoma: A retrospective analysis of 53 cases. Gynecol Oncol 108: 306-311, 2008.

16. Shah JP, Bryant CS, Kumar S, Ali-Fehmi R, Malone JM Jr and Morris RT: Lymphadenectomy and ovarian preservation in low-grade endometrial stromal sarcoma. Obstet Gynecol 112: $1102-1108,2008$

17. Reich $\mathrm{O}$ and Regauer S: Aromatase expression in low-grade endometrial stromal sarcomas: An immunohistochemical study. Mod Pathol 17: 104-108, 2004.

18. Ushijima K, Yahata H, Yoshikawa H, Konishi I, Yasugi T, Saito T, Nakanishi T, Sasaki H, Saji F, Iwasaka T, et al: Multicenter phase II study of fertility-sparing treatment with medroxyprogesterone acetate for endometrial carcinoma and atypical hyperplasia in young women. J Clin Oncol 25: 2798-1803, 2007. 